\title{
Uma contribuição para a avaliação da Atenção Primária à Saúde pela perspectiva do usuário
}

\section{I ${ }^{1}$ Marcele Bocater Paulo de Paiva, ${ }^{2}$ Walter Mendes, \\ ${ }^{3}$ Ana Laura Brandão, ${ }^{4}$ Carlos Eduardo Aguilera Campos I}

Resumo: A avaliação pela perspectiva do usuário é uma parte essencial das avaliações dos serviços de saúde, tendo, entre outras, a finalidade oferecer subsídios para a melhoria da qualidade do cuidado ofertado. A polissemia conceitual e metodológica sobre esse tema é grande e exige esforços de maior teorização. O presente estudo objetiva contribuir para o desenvolvimento de uma metodologia para avaliar, na perspectiva do usuário, a qualidade dos serviços prestados pela Atenção Primária à Saúde (APS). Nesse sentido, foi feito um levantamento na literatura das dimensôes, dos indicadores e de nove instrumentos de avaliação pela perspectiva do usuário, os quais, posteriormente, foram submetidos a julgamento de especialistas, usando-se a técnica Delphi. Houve consenso entre os especialistas sobre 14 dimensões e 56 elementos de mensuração. As dimensões foram julgadas por grau de relevância, sendo consideradas mais relevantes: acesso/ acessibilidade/disponibilidade; relação profissionalusuário; e informação. Traçou-se um panorama sobre metodologias de avaliação pela perspectiva do usuário, além de um arcabouço para a construção de um ou mais instrumentos de avaliação, cujo tamanho possa ser controlado com base na relevância dos itens.

> Palavras-chave: satisfação do usuário; serviços de saúde; avaliação em saúde; Técnica Delphi.

\author{
${ }^{1}$ Universidade do Estado do Rio \\ de Janeiro. Rio de Janeiro-RJ, \\ Brasil. Endereço eletrônico: \\ marcelepaiva@gmail.com \\ ${ }^{2}$ Escola Nacional de Saúde \\ Pública Sergio Arouca / Fiocruz. \\ Rio de Janeiro-RJ, Brasil. \\ Endereço eletrônico: walter@ \\ ensp.fiocruz.br \\ ${ }^{3}$ Escola Nacional de Saúde \\ Pública Sergio Arouca / \\ Fiocruz. Rio de Janeiro-RJ, \\ Brasil. Endereço eletrônico: \\ alaurabrandao@gmail.com \\ ${ }^{4}$ Universidade Federal do Rio de \\ Janeiro, Medicina de Família e \\ da Comunidade. Rio de Janeiro- \\ RJ, Brasil. Endereço eletrônico: \\ cadu@medicina.ufrj.br
}

Recebido em: 06/11/2014 Aprovado em: 09/03/2015 


\section{Introdução}

A satisfação do usuário com o serviço de saúde é um dos resultados desejados do cuidado (DONABEDIAN, 1990). Usuários satisfeitos aderem mais ao tratamento e às orientações prescritas (SILVA; FORMIGLI, 1994) e oferecem mais informaçôes ao cuidador, o que influencia positivamente o desfecho terapêutico e melhora sua qualidade de vida (ESPERIDIÃO; TRAD, 2006). A avaliação da satisfação do usuário constitui um importante indicador para estimar a qualidade dos serviços, sendo, na verdade, uma das principais razóes para se trabalhar com a melhoria da qualidade.

A avaliação na perspectiva do usuário iniciou-se com a perspectiva de aumentar a adesão dos usuários ao tratamento prescrito por profissionais de saúde e, posteriormente, passou a integrar os programas de melhoria de qualidade dos serviços de saúde (WARE; SNYDER, 1975).

Esse tipo de avaliação só se difundiu no Brasil na segunda metade da década de 1990, com o aumento da participação popular nos destinos da saúde (ESPERIDIÃO; TRAD, 2006). Ainda são escassas as avaliações sistemáticas que incluam a perspectiva do usuário, mesmo considerando importante que esta seja institucionalizada nos serviços de saúde (VIEIRA-DA-SILVA, 2005; SILVA; FORMIGLI, 1994).

A avaliação pela perspectiva do usuário contribui para a melhor adequação dos serviços (ESPERIDIÃO; TRAD, 2005), definida como a relação positiva entre o provimento de serviço e as necessidades de saúde (VUORI, 1991). A opinião e o envolvimento dos usuários permitem uma utilização mais adequada dos serviços, com melhores resultados, maior motivação e menos reclamações (SANTOS et al., 2007).

Esse tipo de avaliação funciona como preditor da adesão ao tratamento, sendo útil para apreciar aspectos de acesso e comunicação, e apoia a tomada de decisão em métodos de organização ou para o provimento do cuidado (FITZPATRICK, 1991), além de poder ser ferramenta importante de empoderamento da população, pois lhe dá voz e possibilita um maior controle social.

Outro fator importante para a realização desse tipo de estudo é a dificuldade de desenho de um instrumento avaliativo destinado à perspectiva 
dos usuários que possa ser adotado como padrão ouro e que seja condizente com a diversidade de contextos geográficos, políticos, organizacionais e econômicos de um país continental como o Brasil. O que ocorre muitas vezes é a importação de instrumentos de diversos países, os quais frequentemente se mostram inadequados à nossa realidade.

De acordo com a literatura científica, verifica-se a existência de uma polissemia conceitual e metodológica sobre o tema. Este trabalho procurou investigar as dimensões e os elementos de mensuração mais relevantes, na opinião de especialistas, com o objetivo de contribuir para aperfeiçoar o método e os instrumentos de avaliação da qualidade dos serviços de saúde na perspectiva do usuário, na Estratégia Saúde da Família (ESF).

\section{Metodologia}

O presente artigo é oriundo de pesquisa metodológica que visa contribuir com o desenvolvimentodeinstrumentosavaliativosdaqualidadedosserviçosprestadospela ESF na perspectiva do usuário. As dimensões e indicadores utilizados foram obtidos por meio de revisão de literatura não sistemática, e o método Delphi foi usado para adequação da proposta.

Para conhecer as dimensões e os elementos de mensuração mais empregados em avaliações pela perspectiva do usuário, foi realizada uma revisão não sistemática da literatura no período de 2005 a 2012, utilizando os descritores patient satisfaction, user satisfaction, health services, evaluation, methodology e review, combinados de formas diferentes. As bases de dados consultadas foram Medline, Web of Science e Lilacs. Os descritores e as bases de dados foram selecionados a partir do levantamento da literatura e, principalmente, da revisão realizada por Esperidião e Trad (2005) sobre a metodologia de avaliação da satisfação de usuários com serviços de saúde, que contemplou o período de 1970 a 2005.

A partir desse levantamento, buscou-se encontrar na literatura autores que tenham construído instrumentos de avaliação. O quadro a seguir apresenta os resultados encontrados, segundo objetivo e público-alvo da avaliação sobre a qualidade do cuidado prestado. 
Quadro 1. Instrumentos da avaliação da satisfação do usuário por país de origem, autoria, objetivo e tipo de respondente

\begin{tabular}{|c|c|c|c|c|}
\hline Instrumentos & $\begin{array}{l}\text { País de } \\
\text { origem }\end{array}$ & Autoria & Objetivo & $\begin{array}{c}\text { Tipo de } \\
\text { respondente }\end{array}$ \\
\hline $\begin{array}{l}\text { Primary Care } \\
\text { Assessment Tool } \\
\text { (PCATool) }\end{array}$ & $\begin{array}{l}\text { Estados } \\
\text { Unidos }\end{array}$ & The Johns Hopkins & $\begin{array}{l}\text { Avaliar a presença de } \\
\text { atributos primários e } \\
\text { derivados da APS }\end{array}$ & $\begin{array}{l}\text { Gestores, } \\
\text { profissionais e } \\
\text { usuários }\end{array}$ \\
\hline $\begin{array}{l}\text { Programa de } \\
\text { Melhoria do } \\
\text { Acesso e da } \\
\text { Qualidade } \\
\text { na Atenção } \\
\text { Básica (PMAQ) }\end{array}$ & Brasil & $\begin{array}{l}\text { Ministério da Saúde } \\
\text { (DAB) }\end{array}$ & $\begin{array}{l}\text { Ampliar o acesso e a } \\
\text { qualidade }\end{array}$ & $\begin{array}{l}\text { Equipes de Saúde } \\
\text { da família, NASF, } \\
\text { CEO e usuários }\end{array}$ \\
\hline $\begin{array}{l}\text { Estudos de } \\
\text { Linha de Base } \\
\text { (ELB) }\end{array}$ & Brasil & $\begin{array}{l}\text { Ministério da } \\
\text { Saúde (DAB, } \\
\text { CAA), Proesf - } \\
\text { componente } 3\end{array}$ & $\begin{array}{l}\text { Criar um referencial } \\
\text { (linha de base) para } \\
\text { posteriores estudos } \\
\text { sobre a qualidade do } \\
\text { cuidado }\end{array}$ & $\begin{array}{l}\text { Gestores, } \\
\text { profissionais e } \\
\text { usuários do Saúde } \\
\text { da Família }\end{array}$ \\
\hline $\begin{array}{l}\text { Barómetro } \\
\text { Sanitario }\end{array}$ & Espanha & $\begin{array}{l}\text { Ministerio } \\
\text { de Sanidad y } \\
\text { Política Social da } \\
\text { Espanha, Centro } \\
\text { de Investigaciones } \\
\text { Sociológicas (CIS) }\end{array}$ & $\begin{array}{l}\text { Avaliar a percepção } \\
\text { dos cidadãos sobre } \\
\text { o funcionamento do } \\
\text { sistema de saúde, sobre } \\
\text { o impacto de medidas } \\
\text { governamentais e } \\
\text { evolução temporal, } \\
\text { avaliar o grau de } \\
\text { penetração das } \\
\text { campanhas de } \\
\text { informação, conhecer } \\
\text { a atitude da maioria } \\
\text { dos cidadãos diante de } \\
\text { problemas de saúde de } \\
\text { interesse específico, } \\
\text { entre outros }\end{array}$ & Usuários \\
\hline $\begin{array}{l}\text { Quality of Care } \\
\text { through patients } \\
\text { eyes (Quote) }\end{array}$ & Alemanha & Quote & $\begin{array}{l}\text { Avaliar a qualidade da } \\
\text { atenção primária }\end{array}$ & $\begin{array}{l}\text { Usuários/pacientes } \\
\text { de determinados } \\
\text { grupos (Quote- } \\
\text { elderly ou Quote- } \\
\text { reumathism) }\end{array}$ \\
\hline
\end{tabular}




\begin{tabular}{|c|c|c|c|c|}
\hline Instrumentos & $\begin{array}{l}\text { País de } \\
\text { origem }\end{array}$ & Autoria & Objetivo & $\begin{array}{l}\text { Tipo de } \\
\text { respondente }\end{array}$ \\
\hline $\begin{array}{l}\text { Programa } \\
\text { Nacional de } \\
\text { Avaliação dos } \\
\text { Serviços de } \\
\text { Saúde }\end{array}$ & Brasil & $\begin{array}{l}\text { Ministério da Saúde } \\
\text {-SAA }\end{array}$ & $\begin{array}{l}\text { Avaliar a qualidade } \\
\text { dos serviços de saúde }\end{array}$ & $\begin{array}{l}\text { Usuários, gestores e } \\
\text { profissionais }\end{array}$ \\
\hline $\begin{array}{l}\text { IMD - } \\
\text { Instrumento } \\
\text { de Melhoria do } \\
\text { Desempenho; } \\
\text { Toques - Técnica } \\
\text { para Orientar } \\
\text { a Qualidade e } \\
\text { Eficiência dos } \\
\text { Serviços de } \\
\text { Saúde }\end{array}$ & Brasil & $\begin{array}{l}\text { Secretaria Estadual } \\
\text { de Saúde do Ceará }\end{array}$ & $\begin{array}{l}\text { Contribuir para } \\
\text { a participação do } \\
\text { usuário na avaliação } \\
\text { da oferta e qualidade } \\
\text { dos serviços de } \\
\text { saúde; melhoria } \\
\text { do desempenho do } \\
\text { serviço }\end{array}$ & $\begin{array}{l}\text { Usuários e } \\
\text { profissionais }\end{array}$ \\
\hline $\begin{array}{l}\text { European Task } \\
\text { Force on Patient } \\
\text { Evaluation of } \\
\text { General Practice } \\
\text { Care (Europep) }\end{array}$ & $\begin{array}{l}\text { Grupo } \\
\text { multicêntrico } \\
\text { composto } \\
\text { por países da } \\
\text { Europa }\end{array}$ & $\begin{array}{l}\text { Grupo de médicos } \\
\text { generalistas e } \\
\text { pesquisadores }\end{array}$ & $\begin{array}{l}\text { Avaliar pela } \\
\text { perspectiva dos } \\
\text { usuários o cuidado } \\
\text { recebido no } \\
\text { atendimento realizado } \\
\text { pelo generalista } \\
\text { sobre determinados } \\
\text { aspectos. }\end{array}$ & Usuários \\
\hline $\begin{array}{l}\text { Pesquisa } \\
\text { Mundial da } \\
\text { Saúde }\end{array}$ & $\begin{array}{l}\text { Vários países } \\
\text { (OMS) }\end{array}$ & $\begin{array}{l}\text { OMS; Fiocruz } \\
\text { (coordenação) }\end{array}$ & $\begin{array}{l}\text { Diagnosticar o } \\
\text { desempenho do } \\
\text { SUS; diagnosticar o } \\
\text { estado de saúde da } \\
\text { população brasileira } \\
\text { e avaliar o cuidado } \\
\text { recebido de acordo } \\
\text { com as expectativas da } \\
\text { população usuária }\end{array}$ & Usuários \\
\hline $\begin{array}{l}\text { Grandes } \\
\text { Centros } \\
\text { Urbanos }\end{array}$ & Brasil & $\begin{array}{l}\text { Ministério da Saúde } \\
\text { - Fiocruz }\end{array}$ & $\begin{array}{l}\text { Analisar a } \\
\text { implementação da } \\
\text { ESF; estabelecimento } \\
\text { de vínculo com a } \\
\text { comunidade e a } \\
\text { conversão do modelo } \\
\text { assistencial }\end{array}$ & $\begin{array}{l}\text { Profissionais da } \\
\text { ESF/usuários/ } \\
\text { gestores/ } \\
\text { conselheiros } \\
\text { municipais }\end{array}$ \\
\hline
\end{tabular}

Fonte: Paiva (2013). 

serviços de APS que mais usualmente eram utilizadas na avaliação por usuários. $\mathrm{O}$ quadro a seguir traz as definições das dimensões encontradas:

\section{Quadro 2. Dimensões da satisfação dos usuários e definiçôes}

\begin{tabular}{|c|c|}
\hline Dimensōes & Definiçãao \\
\hline $\begin{array}{l}\text { 1. Continuidade do } \\
\text { cuidado dentro do } \\
\text { sistema de saúdel } \\
\text { coordenaçáo }\end{array}$ & $\begin{array}{l}\text { Capacidade do sistema de saúde de prestar serviços de forma ininterrupta e } \\
\text { coordenada entre diferentes níveis de atenção (PROADESS, 2012). } \\
\text { Integração de todos os serviços relacionados à saúde, onde quer que tenham } \\
\text { sido recebidos (STARFIELD, 2002). }\end{array}$ \\
\hline $\begin{array}{l}\text { 2. Continuidade } \\
\text { do cuidado com o } \\
\text { mesmo profissional } \\
\text { / longitudinalidade }\end{array}$ & $\begin{array}{l}\text { Quando os pacientes se consultam com o mesmo profissional ou visitam a } \\
\text { mesma unidade de saúde entre uma consulta e outra, ou mesmo em determinado } \\
\text { período de tempo, estabelecendo uma relação pessoal entre profissionais e } \\
\text { usuários de longa duração (STARFIELD, 2002). }\end{array}$ \\
\hline 3. Informação & $\begin{array}{l}\text { O profissional de saúde deve ouvir o paciente cuidadosamente e fornecer } \\
\text { explicaçóes de modo que ele possa entender, dispondo de tempo suficiente } \\
\text { para esclarecer todas as suas dúvidas (OMS, } 2000 \text { apud VAITSMAN; } \\
\text { ANDRADE, 2005). } \\
\text { Fornecimento de informaçóes relevantes para o paciente, estímulo ao } \\
\text { autocuidado, atenção para problemas psicológicos dos pacientes, suporte aos } \\
\text { parentes dos pacientes (WENSING et al., 1998). }\end{array}$ \\
\hline $\begin{array}{l}\text { 4. Relação } \\
\text { profissional-usuário }\end{array}$ & $\begin{array}{l}\text { Modo como os prestadores interagem pessoalmente com os pacientes (exemplos: } \\
\text { respeito, amizade e cortesia) (WARE et al., } 1983 \text { apud SITZIA; WOOD, 1997). } \\
\text { Respeito e consideração, escuta, compreensão, respeito às decisões, acolhida, } \\
\text { gentileza por parte dos profissionais da equipe, respeito à privacidade, } \\
\text { confidencialidade e discrição (PRÉVOST; FAFARD; NADEAU, 1988; } \\
\text { TRAD et al., 2002). }\end{array}$ \\
\hline $\begin{array}{l}\text { 5. Segurança do } \\
\text { paciente }\end{array}$ & $\begin{array}{l}\text { Capacidade do sistema de saúde de identificar, evitar ou minimizar os riscos } \\
\text { potenciais das intervenções em saúde ou ambientais (PROADESS, 2012). }\end{array}$ \\
\hline $\begin{array}{l}\text { 6. Acesso / } \\
\text { disponibilidade / } \\
\text { acessibilidade }\end{array}$ & $\begin{array}{l}\text { Grau de ajuste entre clientes e o sistema de saúde, numa interpretação da } \\
\text { ideia, na relação entre a oferta e os indivíduos (PENCHANSKY; THOMAS, } \\
\text { 1981). Compõem o conceito de acesso: disponibilidade de serviços (volume e } \\
\text { tipo) em relação às necessidades; acessibilidade, caracterizada pela adequação } \\
\text { entre a distribuição geográfica dos serviços e dos pacientes; e acolhimento } \\
\text { (accomodation), que representa a relação entre a forma como os serviços } \\
\text { organizam-se para receber os clientes e a capacidade de os clientes se adaptarem } \\
\text { a essa organização (PENCHANSKY; THOMAS, 1981). } \\
\text { Capacidade das pessoas de obter os serviços necessários no lugar e momento } \\
\text { certos (PROADESS, 2012). }\end{array}$ \\
\hline
\end{tabular}




\begin{tabular}{|c|c|}
\hline Dimensōes & Definiçãao \\
\hline $\begin{array}{l}\text { 7. Organização / } \\
\text { estrutura }\end{array}$ & $\begin{array}{l}\text { Questões da parte administrativa e da relação do usuário com o serviço de } \\
\text { saúde que podem ser afetadas pelo funcionamento do mesmo e elementos do } \\
\text { ambiente físico relacionados com o processo de atenção (SERRANO-DEL- } \\
\text { ROSAL; LORIENTE-ARIN, 2008). } \\
\text { Características do ambiente em que o cuidado é ofertado (exemplos: sinalização, } \\
\text { estado de conservação e limpeza das instalações físicas, atmosfera agradável) } \\
\text { (WARE et al., } 1983 \text { apud SITZIA; WOOD, 1997). }\end{array}$ \\
\hline 8. Vínculo & $\begin{array}{l}\text { Estabelecimento, ao longo do tempo, de relaçôes de confiança e afetividade } \\
\text { entre o usuário e o profissional, garantindo a corresponsabilização pela saúde, } \\
\text { além de possuir potencial terapêutico (BRASIL, 2012). }\end{array}$ \\
\hline $\begin{array}{l}\text { 9. Direito dos } \\
\text { pacientes }\end{array}$ & $\begin{array}{l}\text { Capacidade do sistema de saúde de assegurar que os serviços respeitem o } \\
\text { indivíduo e a comunidade e estejam orientados às pessoas (PROADESS, 2012). }\end{array}$ \\
\hline $\begin{array}{l}\text { 10. Confiabilidade / } \\
\text { expectativa }\end{array}$ & $\begin{array}{l}\text { Capacidade de os serviços cumprirem o que prometeram e estarem habilitados } \\
\text { para realizar o serviço (PARASURAMAN; ZEITHAML; BERRY, 1988). }\end{array}$ \\
\hline $\begin{array}{l}\text { 11. Qualidade } \\
\text { técnica / efetividade } \\
\text { / resolutividade }\end{array}$ & $\begin{array}{l}\text { Estimular práticas resolutivas, racionalizar e adequar o uso de medicamentos } \\
\text { eliminando ações desnecessárias (PNH, } 2002 \text { apud VAITSMAN; } \\
\text { ANDRADE, 2005). } \\
\text { Nível de aplicação da tecnologia no cuidado de saúde e conhecimento médio } \\
\text { disponível correntemente (inclui continuidade e satisfação do consumidor) } \\
\text { (VUORI, 1991, p. 37). }\end{array}$ \\
\hline 12. Integralidade & $\begin{array}{l}\text { Refere-se à existência de uma ampla gama de serviços na APS, buscando } \\
\text { responder às mais variadas necessidades em saúde. Abrange a promoção e } \\
\text { a proteção da saúde, a prevenção de agravos, o diagnóstico, o tratamento, a } \\
\text { reabilitação e a manutenção da saúde (STARFIELD, 2002). }\end{array}$ \\
\hline $\begin{array}{l}\text { 13. Orientação } \\
\text { familiar }\end{array}$ & $\begin{array}{l}\text { Centrada na família; conhecimento das famílias, dos seus membros e } \\
\text { fatores familiares relacionados à gênese e ao tratamento das doenças } \\
\text { (STARFIELD, 2002). }\end{array}$ \\
\hline $\begin{array}{l}\text { 14. Orientação } \\
\text { comunitária }\end{array}$ & $\begin{array}{l}\text { Engloba o conhecimento das necessidades de saúde da população, determinantes } \\
\text { da saúde e potencialidades da mesma, bem com o envolvimento comunitário da } \\
\text { unidade (STARFIELD, 2002). }\end{array}$ \\
\hline
\end{tabular}

Fonte: Paiva (2013).

$\mathrm{O}$ número de elementos de mensuração dos instrumentos variou bastante. Enquanto o PCATool (versão adulto) elenca 55 questões, o Europep propõe, a partir de 2006, uma versão de 23 questões, o Quote possui dez questôes e o PNASS tem 14 questões para usuários não internados (BRASIL, 2005, 2010; 
KERSSENS et al., 2004; GROL et al., 2000). A partir dos dez instrumentos descritos na literatura, foram encontrados 112 elementos de mensuração, após exclusão das variáveis de mesmo significado. Esses elementos foram classificados segundo as 14 dimensões e instrumentos de origem.

O método Delphi foi utilizado para identificar, segundo a opinião de especialistas, as dimensóes e os elementos de mensuração que deveriam compor uma metodologia de avaliação da ESF na perspectiva do usuário. Essa técnica busca opinióes coletivas de especialistas a respeito de determinado evento, visando, portanto, estabelecer um consenso de um grupo bem qualificado tecnicamente (WRIGHT; GIOVINAZZO, 2000). Suas características essenciais são as trocas de informações e opiniōes entre os respondentes, o anonimato das respostas e a possibilidade de revisão de visões individuais sobre determinado evento com base em uma representação estatística da visão do grupo (WRIGHT; GIOVINAZZO, 2000). Uchoa et al. (2008) citam que o caráter participativo e flexível do método Delphi tem papel de relevância em avaliações de quinta geração, ou avaliações emancipatórias (empowerment evaluations).

Outras metodologias também poderiam ser empregadas, tais como a realização de oficinas e grupos focais. Entretanto, houve limitação e impossibilidade da presença de todos os participantes e especialistas em um mesmo local e horário. O método Delphi, com questionário em formato eletrônico, mostrou-se mais factível.

Assim, um questionário eletrônico estruturado, com as dimensões, as definições e os elementos de mensuração, foi circulado por duas rodadas entre os especialistas para a formação de um grau de convergência das respostas (WRIGHT; GIOVINAZZO, 2000; SOUZA; VIEIRA-DA-SILVA; HARTZ, 2005). Os especialistas foram contatados por mensagem eletrônica e utilizou-se o aplicativo Google Docs para elaborar e disseminar o questionário.

Os especialistas foram convidados a julgar as dimensões da avaliação de serviços de APS pela perspectiva do usuário, com base numa escala de 1 a 9, desde relevância mínima até relevância máxima. Eles também avaliaram se os elementos de mensuração pertenciam à dimensão em que estavam previamente classificados e, em caso afirmativo, solicitava-se que julgassem a validade dos 
mesmos, ou seja, se realmente aferiam o que fora proposto (FIGUEIRÓ; FRIAS; NAVARRO, 2010).

Em cada rodada, foi disponibilizado um espaço para respostas qualitativas, como comentários sobre as questões ou cenários não contemplados, que foram sistematizadas e reenviadas com o questionário nas rodadas seguintes (SOUZA; VIEIRA-DA-SILVA; HARTZ, 2005).

A técnica Delphi teve duração de dois meses e meio, sendo de um mês o intervalo para os especialistas responderem à primeira rodada, 15 dias para a sistematização dos dados a serem enviados novamente aos especialistas, e mais um mês de intervalo para a segunda rodada.

Considerou-se um especialista a pessoa que tivesse "profundo conhecimento do assunto, seja por formação acadêmica ou por experiência na área de atuação que foi avaliada” (WRIGHT; GIOVINAZZO, 2000). Para selecioná-los, estabeleceram-se os seguintes critérios: médicos com título de especialista em medicina de família e comunidade ou residência médica; enfermeiros com especialização ou residência em saúde da família - ambos com mais de dois anos de experiência profissional na ESF —; pesquisadores em avaliação de qualidade em APS; e gestores de saúde com experiência em APS.

Informantes-chave (médicos e enfermeiros reconhecidos por seus pares pela qualidade técnica) ajudaram no recrutamento dos médicos e enfermeiros especialistas. Os informantes-chave participam de discussões da Sociedade Brasileira de Medicina de Família \& Comunidade e da Associação de Medicina de Família \& Comunidade do Rio de Janeiro, e alguns são coordenadores da residência de Medicina de Família e Comunidade.

Os pesquisadores em avaliação de qualidade em APS foram selecionados entre os autores dos artigos selecionados na revisão bibliográfica. Os gestores de saúde foram escolhidos entre os que tinham experiência na gestão em APS, exercendo ou não tal função no momento da pesquisa. No total, foram selecionados 30 especialistas.

A metodologia de análise da técnica Delphi foi a seguinte: entre as rodadas de perguntas feitas aos especialistas houve o retorno dos resultados para possibilitar a comparação da resposta individual com a coletiva. $\mathrm{Na}$ segunda rodada, os especialistas receberam o resultado da primeira para que pudessem comparar seu 
ponto de vista com a média de opinião anônima dos demais especialistas, assim como uma síntese da pesquisa, do método, de seus objetivos e do propósito de uma segunda rodada.

Sobre as dimensões:

- notas menores ou iguais a 3 e com percentual de concordância igual ou superior a $75 \%$ foram consideradas de baixa relevância e descartadas;

- notas maiores ou iguais a 7 e com percentual de concordância igual ou superior a $75 \%$ foram consideradas de elevada relevância.

Em ambos os casos, a pergunta julgada pertinente não foi levada para a segunda rodada. Os demais casos, considerados "sem consenso" foram levados para a segunda rodada.

Em relação a questões éticas, foi enviada a cada pesquisador uma carta com os objetivos e a metodologia da pesquisa, o referencial teórico utilizado, um convite à participação na pesquisa e um termo de consentimento de livre consentimento. A resposta do questionário via Internet significava o aceite do termo do Comitê de Ética em Pesquisa. O projeto de pesquisa foi aprovado pelo Comitê de Ética em Pesquisa da Escola Nacional de Saúde Pública - Fundação Oswaldo Cruz (número CAAE: 028141127000.5240).

\section{Resultados}

As respostas dos especialistas foram recebidas em duas rodadas. Na primeira, 56\% (17 de 30) especialistas responderam o questionário até 30 dias após ter sido enviado. $\mathrm{Na}$ segunda rodada, $76 \%$ (13 de 17) especialistas enviaram suas respostas, também em um período máximo de 30 dias. $\mathrm{O}$ percentual de abstenção mostrou-se coerente com o descrito na literatura, segundo Wright e Giovinazzo (2000), obtendo abstenção de $44 \%$ na primeira rodada e $24 \%$ na segunda rodada).

As dimensōes foram, então, ranqueadas segundo seu grau de relevância e apresentadas com os resultados e suas definições escolhidas na literatura. A nota média de relevância e o percentual de concordância sobre as dimensões são apresentados na tabela a seguir: 
Tabela 1. Nota média conferida pelos especialistas e grau de concordância, segundo

dimensôes

\begin{tabular}{|l|l|l|}
\hline \multicolumn{1}{|c|}{ Dimensões } & Nota média & $\begin{array}{c}\text { Grau de } \\
\text { concordância (\%) }\end{array}$ \\
\hline Acesso/acessibilidade/disponibilidade & 8,82 & 100,0 \\
\hline Relação profissional-usuário & 8,64 & 100,0 \\
\hline Informação & 8,47 & 94,1 \\
\hline $\begin{array}{l}\text { Continuidade do cuidado dentro do sistema/ } \\
\text { coordenação }\end{array}$ & 8,05 & 88,2 \\
\hline $\begin{array}{l}\text { Continuidade do cuidado com o mesmo } \\
\text { profissional/longitudinalidade }\end{array}$ & 8,00 & 94,1 \\
\hline Integralidade & 8,00 & 88,2 \\
\hline Vínculo & 7,88 & 94,1 \\
\hline Direito do paciente & 7,88 & 82,4 \\
\hline Segurança do paciente & 7,70 & 76,5 \\
\hline Qualidade técnica/efetividade/resolutividade & 7,64 & 82,4 \\
\hline Organização/estrutura & 7,70 & 82,4 \\
\hline Confiabilidade/expectativa & 7,29 & 64,7 \\
\hline Orientação comunitária & 7,23 & 70,6 \\
\hline Orientação familiar & 7,23 & 70,6 \\
\hline
\end{tabular}

Fonte: Paiva (2013).

As três dimensões com maior grau de relevância foram acesso, relação profissional-usuário e informação, enquanto as com menor grau de relevância corresponderam a confiabilidade/expectativa, orientação comunitária e orientação familiar.

$\mathrm{Na}$ resposta aberta, diversos especialistas consideraram que vínculo e relação profissional-usuário deveriam fundir-se em uma única dimensão. Alguns indicaram a possibilidade de unir as dimensóes direito do paciente, segurança do paciente e confiabilidade. 
Considerando-se os 112 elementos de mensuração elencados, para 64 houve concordância entre os especialistas, tanto de que pertenciam à dimensão proposta quanto de que eram válidos para aquela dimensão. Assim, 57\% dos elementos foram julgados válidos e pertencentes à dimensão citada.

38 elementos de mensuração quanto ao pertencimento à dimensão citada não obtiveram consenso entre os especialistas, mas foram indicados como passíveis de classificação em outra dimensão. Assim, na segunda rodada, os especialistas foram solicitados a julgar os indicadores de acordo com a nova classificação de dimensão. Para dez elementos, houve consenso entre os especialistas de que pertenciam à dimensão, porém não houve consenso para avaliar aquela dimensão.

Figura 1. Número de elementos de mensuração, segundo as fases e obtenção de consenso.

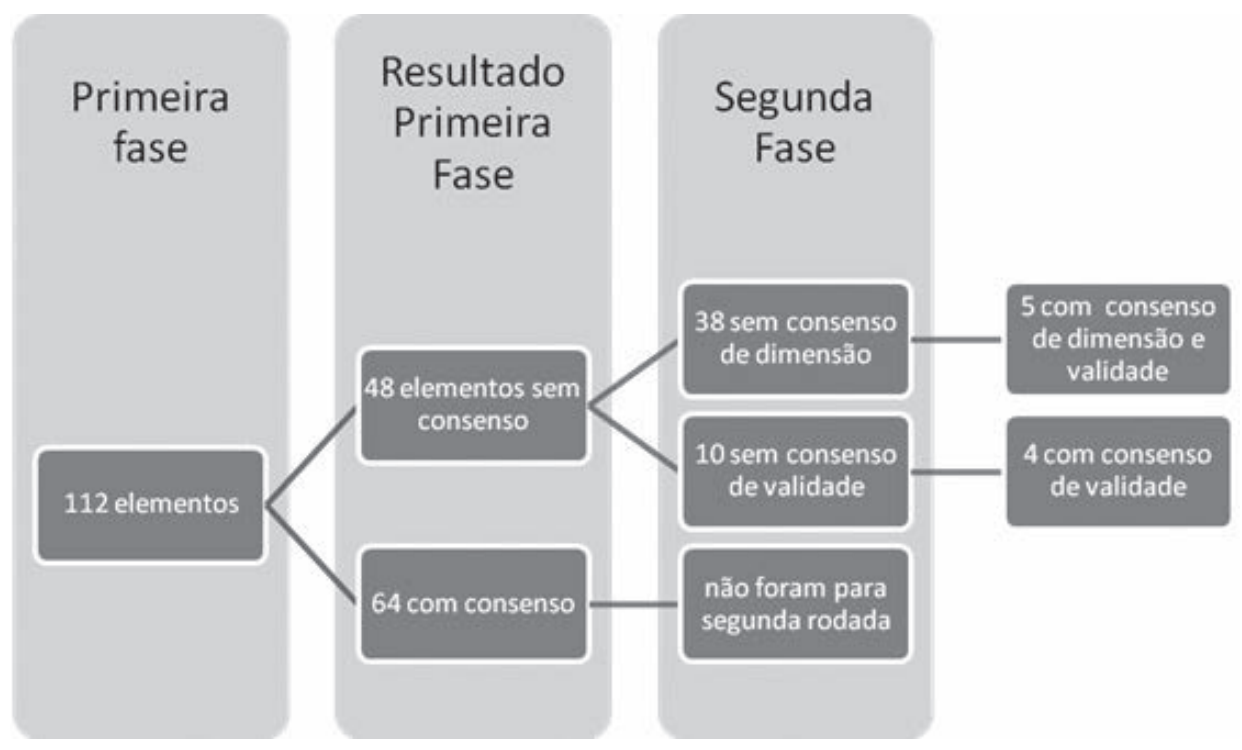

Fonte: Paiva (2013).

$\mathrm{Na}$ segunda rodada, nove elementos de mensuração tiveram julgamentos alterados (cinco em relação à dimensão e quatro no que se refere à validade). Os comentários mais relevantes foram:

- possibilidade de agregar ainda mais os elementos de mensuração relacionados ao tempo de espera da consulta/atendimento e à estrutura física; 
- ressalvas quanto à dimensão vínculo e relação profissional-usuário, longitudinalidade e continuidade de cuidado na mesma dimensão. Foi sugerido que se criassem três subdimensões para "continuidade" para informações, atenção e vínculo, e que nesse aspecto se aproximassem, propondo a criação de subdimensões para longitudinalidade;

- a fusão de dois elementos de mensuração que avaliam o mesmo fato pode exigir a reformulação das perguntas e, algumas vezes, um dos elementos pode ser excluído;

- a presença de diferentes tipos de perguntas (abertas, fechadas, enumeração) num mesmo instrumento dificulta a avaliação da validade do instrumento;

- a presença de mais de um elemento aferindo aspectos parecidos da mesma dimensão pode aumentar a confiabilidade do instrumento.

$\mathrm{Na}$ primeira rodada, 64 elementos de mensuração obtiveram consenso, o que constituiria um instrumento longo para autoaplicação, sendo necessária a presença de um entrevistador. Diante disso, o instrumento final (Quadro 3) ficou com 42 elementos.

Quadro 3. Elementos de mensuração selecionados pelos especialistas, segundo as dimensões

\begin{tabular}{|l|l|}
\hline Dimensóes & Elementos de mensuração (instrumento de origem) \\
\hline $\begin{array}{l}\text { Continuidade } \\
\text { do cuidado } \\
\text { dentro do } \\
\text { sistema de saúde/ } \\
\text { coordenação }\end{array}$ & $\begin{array}{l}\text { 1. Quando é necessário, o médico, enfermeiro ou dentista nesse } \\
\text { serviço o envia para uma consulta com o especialista? (PMAQ) } \\
\text { 2. Você demorou até ser atendido pelo especialista na última vez em } \\
\text { 3. Depois de ir ao especialista algum profissional desse serviço } \\
\text { conversou com você sobre a consulta? (PMAQ) }\end{array}$ \\
\hline $\begin{array}{l}\text { Continuidade } \\
\text { do cuidado } \\
\text { com o mesmo } \\
\text { profissional/ } \\
\text { longitudinalidade }\end{array}$ & $\begin{array}{l}\text { 4. O profissional que o atendeu conhece você, sua história e o } \\
\text { seguimento dos seus problemas de saúde? (Barómetro Sanitario) } \\
\text { geralmente vai quando fica doente ou precisa de conselhos sobre a } \\
\text { 6. Hán médico/enfermeiro desse serviço de saúde que o/a conhece } \\
\text { 7. Há um médico/enfermeiro ou serviço de saúde que é mais } \\
\text { responsável por seu atendimento de saúde? (PCAT) }\end{array}$ \\
\hline
\end{tabular}




\begin{tabular}{|c|c|}
\hline Dimensões & Elementos de mensuração (instrumento de origem) \\
\hline Informação & $\begin{array}{l}\text { 8. O profissional que o atendeu explicou sobre os exames e } \\
\text { tratamentos receitados? (Europep) } \\
\text { 9. O profissional que o atendeu informou sobre o que gostaria de } \\
\text { saber sobre seus sintomas e sobre sua doença? (Europep) } \\
\text { 10. Você foi incentivado pelo profissional para seguir as orientações } \\
\text { e tratamento de saúde? (Europep) } \\
\text { 11. Como você classificaria os conselhos do médico sobre } \\
\text { alimentação, exercício, cigarro, álcool, etc., pontuando-os de } 0 \text { a } 10 \text { ? } \\
\text { (Barómetro Sanitario) } \\
\text { 12. Como você classificaria as informaçôes disponíveis para acessar } \\
\text { o especialista ou ingressar no hospital, pontuando-as de } 0 \text { a } 10 \text { ? } \\
\text { (Barómetro Sanitario) } \\
\text { 13. O profissional lhe informou em linguagem compreensível a } \\
\text { respeito dos tratamentos que foram prescritos, para que você possa ter } \\
\text { clareza do seu problema, e perguntou se desejava alguma informação } \\
\text { complementar? (Quote) }\end{array}$ \\
\hline $\begin{array}{l}\text { Relação } \\
\text { profissional - } \\
\text { usuário/vínculo } \\
\text { (unificadas) }\end{array}$ & $\begin{array}{l}\text { 14. Foi permitida sua participação nas decisões do cuidado que o } \\
\text { profissional prestou? (Europep) }\end{array}$ \\
\hline $\begin{array}{l}\text { Segurança do } \\
\text { paciente }\end{array}$ & $\begin{array}{l}\text { 15. Alguém da sua família já sofreu algum tipo de erro grave na } \\
\text { assistência à saúde recebida na atenção primária? (Barómetro Sanitario) } \\
\text { 16. Este erro o afetou de uma maneira: muito grave; bastante grave; } \\
\text { pouco grave; nada grave (Barómetro Sanitario) }\end{array}$ \\
\hline $\begin{array}{l}\text { Acesso/ } \\
\text { disponibilidade/ } \\
\text { acessibilidade }\end{array}$ & $\begin{array}{l}\text { 17. Como você avalia a proximidade desse serviço? (Barómetro } \\
\text { Sanitario) } \\
\text { 18. Como você avalia o tempo de espera até entrar na consulta? } \\
\text { (Barómetro Sanitario) } \\
\text { 19. Como você avalia o tempo que se toma para fazer as provas } \\
\text { diagnósticas? (Barómetro Sanitário) } \\
\text { 20. Como você avalia as condições de acesso físico à unidade de } \\
\text { saúde (rampas, escada, calçadas, identificação visual)? (PMAQ) } \\
\text { 21. Você considera o horário de funcionamento da unidade adequado } \\
\text { às suas necessidades? (PMAQ) }\end{array}$ \\
\hline
\end{tabular}




\begin{tabular}{|c|c|}
\hline Dimensóes & Elementos de mensuração (instrumento de origem) \\
\hline $\begin{array}{l}\text { Acesso/ } \\
\text { disponibilidade/ } \\
\text { acessibilidade }\end{array}$ & $\begin{array}{l}\text { 22. Você conseguiu marcar consulta de rotina nesse serviço? } \\
\text { (PMAQ) } \\
\text { 23. Você conseguiu atendimento nesse serviço quando se sentiu } \\
\text { muito mal (dor, febre, pressão alta)? (PMAQ) } \\
\text { 24. Qual foi o tempo de espera, em média, para conseguir a sua } \\
\text { consulta? (PMAQ) } \\
\text { 25. Você considera que: demorou muito; demorou; demorou pouco; } \\
\text { não demorou (PNASS) } \\
\text { 26. Com esse tempo você se sente: muito satisfeito; satisfeito; } \\
\text { insatisfeito; muito insatisfeito (PNASS) } \\
\text { 27. Quando você necessita de uma consulta de revisão (consulta de } \\
\text { rotina, check-up), você vai ao seu médico ou enfermeiro de família } \\
\text { antes de ir a outro serviço de saúde? (PCAT) } \\
\text { 28. Como você considera o acesso a medicamentos em atendimento } \\
\text { realizado pela ESF (Grandes Centros Urbanos)? }\end{array}$ \\
\hline $\begin{array}{l}\text { Organização/ } \\
\text { estrutura }\end{array}$ & $\begin{array}{l}\text { 29. Como você julga a limpeza dos ambientes? (PNASS) } \\
\text { 30. Como você julga o conforto dos ambientes? (PNASS) } \\
\text { 31. O estabelecimento é bem sinalizado para você encontrar o local } \\
\text { onde precisa ir? (PNASS) }\end{array}$ \\
\hline $\begin{array}{l}\text { Direito dos } \\
\text { pacientes }\end{array}$ & $\begin{array}{l}\text { 32. Você possui a liberdade em escolher o profissional de saúde? } \\
\text { (ELB) } \\
\text { 33. Quando não é bem atendido você sabe onde reclamar? (PNASS) } \\
\text { 34. Você é sempre examinado em ambiente fechado? (IMD/Toques) } \\
\text { 35. Quando existe uma terceira pessoa presente durante o } \\
\text { atendimento (por exemplo, estagiário, estudante, médico visitante), } \\
\text { o pessoal do serviço explica essa presença e pede sua permissão? } \\
\text { (IMD/Toques) }\end{array}$ \\
\hline $\begin{array}{l}\text { Confiabilidade/ } \\
\text { expectativa }\end{array}$ & Nenhum elemento de mensuração alcançou consenso para esta dimensão \\
\hline $\begin{array}{l}\text { Qualidade } \\
\text { técnica/ } \\
\text { efetividade/ } \\
\text { resolutividade }\end{array}$ & $\begin{array}{l}\text { 36. Competência e habilidade do profissional de saúde: muito bom; } \\
\text { bom; ruim; muito ruim } \\
\text { 37. Conseguem resolver o problema de saúde neste atendimento? } \\
\text { (Grandes Centros Urbanos) } \\
\text { 38. A equipe buscou resolver sua necessidade de saúde nesse serviço? } \\
\text { (Grandes Centros Urbanos) }\end{array}$ \\
\hline
\end{tabular}




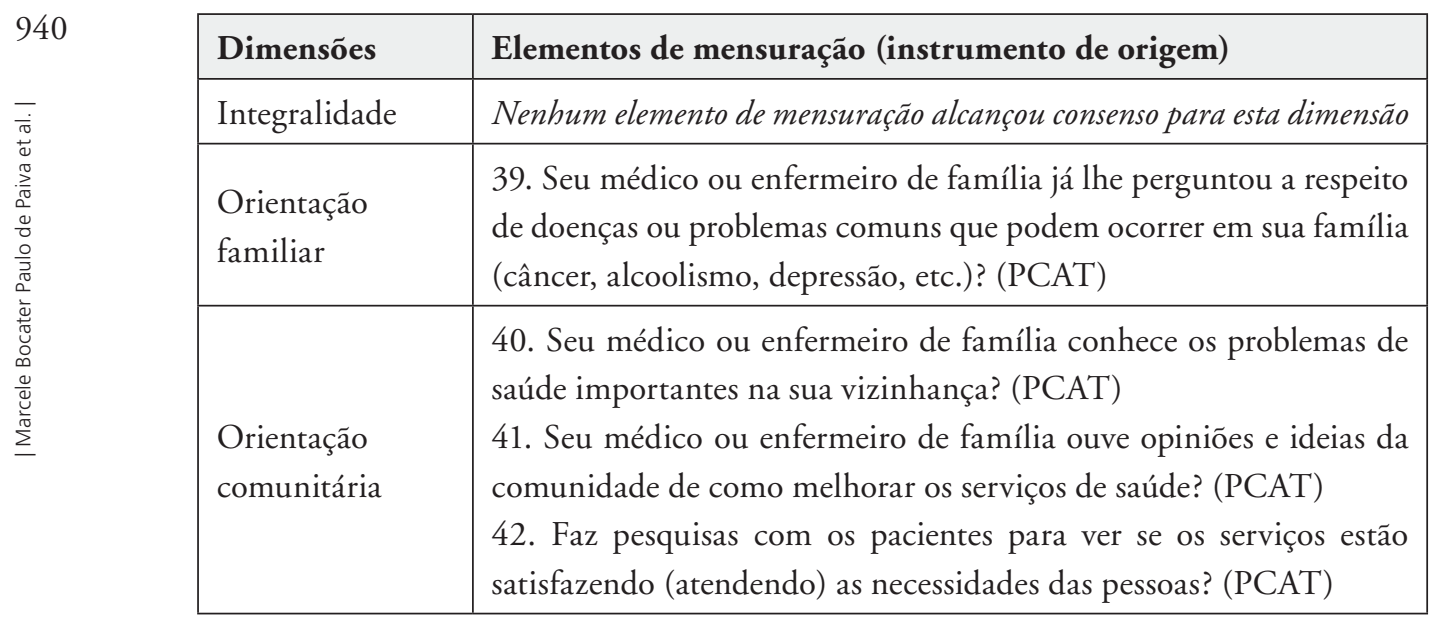

Fonte: Paiva (2013)

\section{Discussão}

Diversos gestores e autores concordam que a busca de instrumento de avaliação factível e reproduzível nacionalmente constitui um desafio (MEDEIROS, 2011). Os especialistas consultados no presente trabalho ressaltaram a importância de introduzir perguntas sobre idade, sexo e condiçōes socioeconômicas dos usuários. Variáveis que parecem ter maior influência na satisfação são idade e escolaridade (SITZIA; WOOD, 1997). A seguir, são discutidos os resultados para cada uma das dimensões avaliadas.

\section{Dimensão acesso}

Esta dimensão é a que possui maior relevância em relação à satisfação do usuário. Tal resultado não foi diferente do encontrado em uma pesquisa que utilizou grupos focais com usuários (MORAES; CAMPOS; BRANDÃO, 2014), sendo também um dos quatro itens mais presentes em estudos sobre a satisfação do usuário (WARE, 1983), o segundo fator com maior significância na avaliação da satisfação dos pacientes (PASCOE, 1993) e um dos atributos principais para avaliação de orientação do sistema para APS (STARFIELD, 2002),

A dimensão acesso/acessibilidade/disponibilidade também se destacou pelo fato de a ESF ser reorientadora e porta de entrada do sistema de saúde (BRASIL, 2012). 
Dimensão relação profissional-usuário

Diversos estudos mostram que a dimensão "relação profissional-usuário" é um dos aspectos essenciais da satisfação do usuário (MISHIMA et al., 2010; ROCHA et al., 2008; COTTA et al., 2005). Ela corresponde a um dos quatro itens mais presentes sobre a satisfação do usuário (WARE, 1983), é uma das dimensões mais bem avaliadas (BRANDÃO; GIOVANELLA; CAMPOS, 2013; ROCHA et al., 2008; TRAD et al., 2002) e, em trabalhos com grupos focais com usuários, também é uma das mais relatadas (MORAES; CAMPOS; BRANDÃO, 2014; TRAD et al., 2002). Essa dimensão está presente no maior número de instrumentos e elementos de mensuração identificados na literatura.

\section{Dimensão informação}

Terceira dimensão com maior relevância no julgamento dos especialistas, sua importância é também destacada pela sua relação com a garantia nos "direitos do paciente", tanto nas cartilhas dos direitos do paciente, quanto nas de humanização e no Código de Ética Médica (VAITSMAN; ANDRADE, 2005). Segundo Esperidião e Trad (2006), essa dimensão pode ser avaliada como parte dos aspectos humanitários da relação médico-paciente. Entretanto, os próprios autores, em outro artigo, reconhecem a autonomia que essa dimensão vem ganhando (ESPERIDIÃO; TRAD, 2005; SITZIA; WOOD, 1997).

Pacientes informados ou educados estão associados a melhores resultados, maior adesão aos tratamentos e diminuição de custo-benefício, com melhor uso dos serviços de saúde e maior satisfação no curto período após a consulta (SITZIA; WOOD, 1997).

\section{Dimensão continuidade do cuidado dentro do sistema de saúde/coordenação}

A coordenação é um atributo primário da APS (STARFIELD, 2002). Essa dimensão está relacionada à função de "filtro dos médicos generalistas" do médico de família e comunidade, que no seu exercício pode coordenar o cuidado em diferentes níveis de atenção (GÉRVAS; FERNANDEZ, 2005; 2006). Em diversos países da Europa, o generalista é o primeiro contato do usuário com o sistema, o que permite, baseado em evidências científicas, encaminhar 
aos especialistas apenas os casos necessários e no momento em que se julgar adequado. Esse papel, entre outras vantagens, possibilita a prevenção quaternária (GÉRVAS; FERNANDEZ, 2005; 2006).

Nos instrumentos internacionais, tais como PCATool, Europep e Barómetro Sanitario, a coordenação apareceu frequentemente como dimensão para avaliação. No trabalho de avaliação de validade e confiabilidade do PCATool-Brasil para população infantil, a dimensão "coordenação" também se mostra presente em cinco dos 45 indicadores usados com validade e confiabilidade apropriadas para uso nacional. Um sinônimo de "coordenação" é "integração de cuidados" (HARZHEIM et al., 2006).

\section{Dimensão continuidade do cuidado com o mesmo profissional/longitudinalidade}

A longitudinalidade constitui um atributo essencial da APS, de que deriva a importância de aferir a orientação à APS pela perspectiva do usuário, além de possibilitar a prevenção quaternária, pela continuidade no atendimento e o conhecimento do paciente (GÉRVAS; FERNANDEZ, 2006).

Segundo revisão feita por Cunha e Giovanella (2011), essa dimensão seria composta por três subdimensões: a continuidade informacional, a relacional, estabelecendo um vínculo duradouro e terapêutico, e a referência da Unidade Básica, prestadora regular de cuidados.

Um dos especialistas sugeriu a possibilidade de agrupá-la à dimensão vínculo. A maioria, porém, considerou esta mais próxima da relação profissional-usuário.

\section{Dimensão segurança do paciente}

Essa foi a oitava dimensão a ser classificada por grau de relevância. Alguns especialistas mencionaram que todas as dimensões são importantes e que atribuíram menos pontos a algumas dimensões para tornar o instrumento factível.

Para esta dimensão, alguns autores referem o "efeito iceberg da segurança do paciente": incidentes registrados com dano ao usuário em consequência do cuidado, com base nos relatórios do Institute of Medicine, são apenas a ponta do iceberg, escondendo a grande quantidade de incidentes sem dano que ocorreram e não afetaram negativamente a vida do paciente (MENDES, 2008; PROADESS, 2012). 
Para a dimensão segurança do paciente foram encontrados apenas dois elementos de mensuração no instrumento do Barómetro Sanitario.

A valoração desta dimensão também coincide com o fato de não terem sido encontrados elementos para sua mensuração em instrumentos brasileiros.

\section{Dimensão direitos dos pacientes}

Introduzida pela OMS em 2000, esta dimensão pode ser dividida em aspectos relacionais: relação profissional-usuário (direito ao sigilo, privacidade, tratamento humanizado, respeito às diferenças, acesso a informações sobre sua doença e tratamento e respeito à autonomia em relação ao tratamento) e relação com os serviços (prestação e infraestrutura). Os elementos de mensuração encontrados para esta dimensão foram retirados de instrumentos brasileiros: IMD/Toques, PNASS e ELB.

Ela foi avaliada em sétimo lugar pelos especialistas, sendo que um deles mencionou que as outras dimensões poderiam ser classificadas como subdimensões de direitos do paciente, como direito a informação e direito a uma relação profissional-usuário humanizada. Porém, nos elementos de mensuração dessas dimensões, existem perguntas sobre como os pacientes percebem o cuidado e sua satisfação em relação a diversos aspectos que as compõem (BRASIL, 2010; ESPANHA, 2011; PROADESS, 2012).

\section{Dimensões orientação familiar e orientação comunitária}

Estas dimensões são atributos derivados da APS (STARFIELD, 2002). Segundo a Organização Mundial dos Médicos de Família, a atividade do médico de família e comunidade deve ser centrada na pessoa, com foco na família, e ser orientada para a comunidade (WONCA, 2002).

Os especialistas apontaram a importância de todas as dimensões; entretanto, estas duas ficaram entre os últimos três lugares.

\section{Dimensão organização/estrutura}

No processo de avaliação da satisfação do usuário existe o viés de gratidão, que corresponde a uma avaliação positiva do usuário unicamente por ter sido atendido. Isso pode aparecer, entre outras, na forma de omissão de críticas ou pela afinidade com o provedor do cuidado (ESPERIDIÃO; TRAD, 2005). 
Tendo em vista que a dimensão organizacional compreende baixíssimos níveis de satisfação nas pesquisas com usuários (TRAD et al., 2002; BRANDÃO; GIOVANELLA; CAMPOS, 2013; MORAES; CAMPOS; BRANDÃO, 2014), seria relevante incluí-la, uma vez que avaliar os pontos de insatisfação no serviço pode ser importante ferramenta para melhoria da qualidade (ESPERIDIÂO; TRAD, 2005; WARE et al., 1983). Entretanto, os especialistas julgaram essa dimensão como a décima por grau de relevância.

\section{Dimensão integralidade}

Também considerada um atributo primário, segundo Starfield (2002), esta dimensão, apesar de ter sido classificada em sexto lugar, não obteve nenhum consenso quanto aos elementos de mensuração. Poucos elementos estavam disponíveis nos instrumentos avaliados para que pudessem ser julgados pelos especialistas. $\mathrm{O}$ único instrumento que dispunha desse item, entre os avaliados, foi o PCATool (BRASIL, 2005, 2010; KERSSENS et al., 2004; GROL et al., 2000).

\section{Dimensão confiabilidade/expectativa}

Foi a dimensão com pior avaliação entre os especialistas. Não houve consenso em nenhuma das rodadas para esta dimensão. Segundo Parasuraman, Zeithaml e Berry (1988), que dividiu a qualidade pela perspectiva do usuário em cinco dimensóes, esta foi a que teve o menor peso comparada às outras quatro dimensões quanto a medidas diretas de importância relativa e peso, coincidindo parcialmente com alguns aspectos do presente estudo.

\section{Conclusão}

A avaliação pela perspectiva do usuário é de relevância, pois inclui a opinião deste como um norteador para o planejamento e a tomada de decisóes nos serviços de saúde. Ela é capaz de mudar as atitudes e decisôes dos provedores de cuidado. Entretanto, existe uma polissemia conceitual e metodológica desse tipo de avaliação.

Apesar da existência de nove instrumentos, apresentados neste artigo, ainda há muitas dificuldades metodológicas para a utilização de um instrumento robusto num país como o Brasil, de dimensões continentais, baixa consciência de cidadania e, em muitos lugares, baixa escolaridade. 
Os esforços de uma avaliação institucionalizada que envolva a perspectiva do usuário vêm crescendo na última década. O presente trabalho caminha em direção a uma maior teorização da metodologia e aponta dimensões e um conjunto de elementos de mensuração para a construção de um novo instrumento, a partir do julgamento de especialistas (método Delphi) dos instrumentos levantados na literatura.

A presente pesquisa traz como contribuição traçar um panorama de metodologias de avaliação dos serviços da ESF pela perspectiva do usuário: o julgamento dos especialistas a respeito dos critérios dos instrumentos existentes na literatura para essa avaliação; apontamentos para construção de um novo instrumento; e um banco de elementos de mensuração para ser usado de acordo com a finalidade do estudo, como avaliação de apenas uma dimensão da satisfação ou das três julgadas mais importantes ou com mais problemas no serviço que se pretende avaliar.

Uma possível limitação do presente trabalho foi a impossibilidade da realização de técnicas de consenso de forma presencial, tendo sido escolhida uma estratégia através de questionário eletrônico.

O último aspecto destacado é a necessidade de incorporação de mais dimensões aos instrumentos de avaliação da ESF pelo usuário, como segurança e direito do paciente. Observa-se, também, a importância da elaboração de alguns elementos de mensuração para dimensões em que ou não houve consenso de elementos de mensuração ou houve consenso de apenas um elemento, tais como "integralidade" e "orientação familiar".

$\mathrm{O}$ estudo chama a atenção para diferentes pontos, entre os quais se destaca a necessidade de continuar construindo uma metodologia robusta de avaliação pela perspectiva do usuário com um instrumento com a possibilidade de aplicabilidade nacional. ${ }^{1}$

\section{Referências}

BRANDÃO, A. L. R. B. S.; GIOVANELLA, L.; CAMPOS, C. E. A. Avaliação da atenção básica pela perspectiva dos usuários: adaptação do instrumento EUROPEP para grandes centros urbanos brasileiros. Ciência \& Saúde Coletiva, v. 18, p. 103-114, 2013.

BRASIL. Ministério da Saúde. Secretaria de Atenção à Saúde. Departamento de Atenção Básica. Coordenação de Acompanhamento e Avaliação. Avaliação na Atenção Básica em Saúde: caminhos da institucionalização. Coordenação técnica do Instituto de Saúde 
Coletiva da Universidade Federal da Bahia e Instituto Materno Infantil Prof. Fernando Figueira, IMIP. Brasília-DF: Ministério da Saúde, 2005.

- Ministério da Saúde. Secretaria de Atenção à Saúde. Departamento de Atenção Básica. Manual do instrumento de avaliação da atenção primária à saúde: primary care assessment tool PCATool-Brasil. Brasília-DF: Ministério da Saúde, 2010.

Autoavaliação para melhoria do acesso e da qualidade da atenção básica. Brasília-DF:

Ministério da Saúde, 2012.

COTTA, R.; M, M. et al. Satisfação dos usuários do Programa de Saúde da Família: avaliando o cuidado em saúde. Scientia Medica, v. 15, n. 4, p. 227, 2005.

CUNHA, E. M.; GIOVANELLA, L. Longitudinalidade/continuidade do cuidado: identificando dimensōes e variáveis para a avaliação da Atenção Primária no contexto do sistema público de saúde brasileiro. Ciência \& Saúde Coletiva, v. 16, supl. 1, p. 1029$1042,2011$.

DONABEDIAN, A. Garantía y monitoria de la calidad de la atención médica: un texto introductorio. México: Instituto Nacional de Salud Pública, 1990.

ESPANHA. Ministerio de Sanidad y Servicios Sociales e Igualdad. Barómetro Sanitario 2011. [Internet]. Madrid: Instituto de Información Sanitária. Disponível em: <http:// www.msssi.gob.es/estadestudios/estadisticas/sisinfsansns/informeanual.htm.>. Acesso em: 26 mar. 2013.

ESPERIDIÃO, M. A.; TRAD, L. A. B. Avaliação da satisfação de usuários. Ciência \& Saude Coletiva, v. 10, supl., p. 303-12, 2005.

. Avaliação de satisfação de usuários: considerações teórico-conceituais. Cadernos de Saúde Pública, v. 22, n. 6, p. 1267-76, 2006.

FIGUEIRÓ, A. C.; FRIAS, P. G.; NAVARRO, L. M. Avaliação em saúde: conceitos básicos para a prática nas instituições. In: SAMICO, I. et al. (Orgs.). Avaliação em saúde: bases conceituais e operacionais. Rio de Janeiro: MedBook, 2010.

FITZPATRICK, R. Surveys of patient satisfaction: important general considerations. British Medical Journal, n. 302, p. 887-889, 1991.

GÉRVAS, J., FERNÁNDEZ, M. La función de filtro del médico general y el papel de los especialistas en los sistemas de salud. Revista Brasileira de Epidemiologia, v. 9, n. 2, 2006.

El fundamento científico de la función de filtro del médico general. Revista Brasileira de Epidemiologia, v. 8, n. 2, p. 205-218, 2005.

GROL, R. et al. Patients in Europe evaluate general practice care: an international comparison. Br J Gen Pract, n. 50, p. 882-887, 2000. 
HARZHEIM, E. et al. Consistência interna e confiabilidade da versão em português do Instrumento de Avaliação da Atenção Primária (PCATool-Brasil) para serviços de saúde infantil. Cadernos de Saúde Pública, v. 22, n. 8, p.1649-1659, 2006.

KERSSENS, J. et al. Comparison of patient evaluations of health care quality in relation to who measures of achievement in 12 European countries. Bull World Health Organ, n. 82, p. 106-114, 2004.

MEDEIROS, F. A. Avaliação da Atenção Primária à Saúde: análise de concordância entre os instrumentos AMQ e PCATool no município de Curitiba, Paraná. Dissertação (Mestrado em Saúde Pública). Universidade Federal do Rio Grande do Sul, Porto Alegre, 2011.

MENDES, E. V. As redes de atenção à saúde. Revista Médica de Minas Gerais, v. 18, n. 4, supl., 2008.

MISHIMA, S. M. et al. A assistência na saúde da família sob a perspectiva dos usuários. Revista Latino Americana de Enfermagem, v. 18, n. 3, p. 148-155, 2010.

MORAES, V. D.; CAMPOS, C. E. A.; BRANDÃO, A. L. R. B. S. Estudo sobre dimensões da avaliação da APS pela perspectiva do usuário. Physis: Revista de Saúde Coletiva. Rio de Janeiro, v. 24, p. 1-2, 2014.

PAIVA, M. B. P. Uma contribuição para um modelo de avaliação da Estratégia da Saúde da Família pela perspectiva do usuário. Dissertação (Mestrado em Saúde Pública) - Escola Nacional de Saúde Pública, Rio de Janeiro, 2013.

PARASURAMAN, A.; ZEITHAML, V.; BERRY, L. A. multiple-item scale for measuring customer perceptions of service quality. Journal of Retailing, p. 12-40, 1988.

PASCOE, G. C. Patient satisfaction in primary health care: a literature review and analysis. Evaluation Program Plan, n. 6, p. 185-210, 1993.

PENCHANSKY, D.; THOMAS, J. The concept of access - definition and relationship to consumer satisfaction. Med Care, n. 19, p. 127-140, 1981.

PRÉVOST, A.; FAFARD, A.; NADEAU, M. La mesure de la satisfaction des usagers dans le domaine de la santé et des services sociaux: l'expérience de la région de la ChaudièreAppalaches. The Canadian Journal of Program Evaluation, v. 1, n. 13, p. 1-23, 1998.

PROADESS. Matriz de dimensões da avaliação de desempenho do sistema de saúde: contexto político, social, econômico e conformação do sistema de saúde. 2012. Disponível em:<http://www.proadess.icict.fiocruz.br/index.php?pag=matp >. Acesso em: 21 set. 2014.

ROCHA, P. M. et al. Avaliação do Programa Saúde da Família em municípios do Nordeste brasileiro: velhos e novos desafios. Cadernos de Saúde Pública, n. 24 (Supl.), p. 69-78, 2008. SANTOS, O. et al. Os centros de saúde em Portugal: a satisfação dos utentes e dos profissionais. Lisboa: Ministério da saúde, Missão para os Cuidados de Saúde Primários, 2007. 
SERRANO-DEL-ROSAL, R.; LORIENTE-ARIN, N. La anatomía de la satisfacción del paciente. Salud Pública de México, v. 50, n. 2, p. 162-172, 2008.

SILVA, L.; FORMIGLI, V. Avaliação em saúde: limites e perspectivas. Cadernos de Saúde Pública, n. 10, p. 80-91, 1994.

SITZIA, J.; WOOD, N. Patient satisfaction: a review of issues and concepts. Social Science Medicine, v. 45, n. 12, p. 1829-1843, 1997.

SOUZA, L. E. P. F.; VIEIRA-DA-SILVA, L. M.; HARTZ, Z. M. A. Conferência de consenso sobre a imagem-objetivo da descentralização da atenção à saúde no Brasil. In: HARTZ, Z. M. A.; VIEIRA-DA-SILVA, L. M. Avaliação em saúde: dos modelos teóricos à prática na avaliação de programas e sistemas de saúde. Rio de Janeiro: Editora Fiocruz, 2005, p. 65-102.

STARFIELD, B. Atenção primária - equilíbrio entre necessidades de saúde, serviços e tecnologia. Brasília: Unesco, Ministério da Saúde, 2002.

TRAD, L.; A.; B. et al. Estudo etnográfico da satisfação do usuário do Programa de Saúde da Família (PSF) na Bahia. Ciência \& Saúde Coletiva, v. 7, n. 3, p. 581-589, 2002.

UCHOA, A. C. et al. técnicas de consenso: potencialidades e limites na avaliação da informação em saúde. In: HARTZ, Z.; FELIZBERTO, E.; SILVA, L. M.; (Org.). Metaavaliação da Atenção Básica em Saúde: teoria e prática. Rio de Janeiro: Editora Fiocruz, 2008, p. 253-282.

VAITSMAN, J.; ANDRADE, G. Satisfação e responsividade: formas de medir a qualidade e humanização da assistência à saúde. Ciência \& Saúde Coletiva, n.10, p. 599-613, 2005.

VIEIRA-DA-SILVA, L. M. Conceitos, abordagens e estratégias para avaliação em saúde In: HARTZ, Z. M. A.; VIEIRA-DA-SILVA, L. M. (Org.). Avaliação em saúde. Dos modelos teóricos à prática na avaliação de Programas e Sistemas de saúde. Rio de Janeiro/Salvador: Editora Fiocruz/EDUFBA, 2005, p. 15-39.

VUORI, H. A qualidade da saúde. Divulgação em Saúde para Debate, n. 3, p. 17-25, 1991.

WARE, J.; et al. Defining and measuring patient satisfaction with medical care. Evaluation and Program Planing, n. 6, p. 247-263, 1983.

WARE, J.; SNYDER, M. Dimensions of patients attitudes regarding doctors and medical care services. Medical Care, n. 13, p. 1-23, 1975.

WENSING, M. et al. A systematic review of the literature on patient priorities for general practice care. Part 1: description of the research domain. Social Science Medicine, v. 47, n. 10, p. 1573-1588, 1998. 
WONCA, A. Definição europeia de medicina geral e familiar (clínica geral / medicina

familiar), 2002. Disponível em: <http://www.woncaeurope.org/sites/default/files/ documents/EuropeanDefinitioninPortuguese.pdf>. Acesso em: 21 set. 2014.

WRIGHT, J. T. C.; GIOVINAZZO, R. A. Delphi: uma ferramenta de apoio ao planejamento participativo. Caderno de Pesquisas em Administração, v. 1, n. 12, p. 64-65, 2000.

\section{Nota}

${ }^{1}$ M. B. P. de Paiva participou redação do artigo, aplicação do instrumento, análise e interpretação dos dados e aprovação final da versão a ser publicada do artigo; W. Mendes participou da orientação e concepção geral do projeto e aprovação final da versão a ser publicada do artigo; A. L. Brandão participou da revisão bibliográfica realizada para o projeto, concepção metodológica do projeto e revisão crítica relevante do conteúdo intelectual, revisão crítica relevante do conteúdo intelectual; C. E. A. Campos participou da concepção geral do projeto e revisão crítica relevante do conteúdo intelectual. 


\section{Abstract}

\section{A contribution to the assessment of the Primary Health Care in the user's viewpoint}

The evaluation seen through the user's perspective is an essential part of evaluations of health services. The final goal is knowing about the user's perspective on the care received and offer subsidies to improve the quality of the service offered. The conceptual and methodological polysemy on this topic is wide and requires great efforts for theorizing. The purpose of this study was to contribute to the development of a methodology to evaluate, through the user's perspective, the quality of services provided by the Primary Care. This way, a review about the dimensions and indicators and nine instruments of evaluation of user's perspective. Then, they were subjected to expert judgment using the Delphi technique. Experts agreed on 14 dimensions and 56 measurement elements. The dimensions were judged by degrees of relevance, being considered as most relevant: access/accessibility / availability, professionaluser relationship and information. An overview of the evaluation of methodologies, through the user's perspective was made, as well as a framework for the construction of one or more instruments of evaluation in which the size of the instrument could be controlled based on the relevance of the items.

Key words: patient satisfaction; user satisfaction; health services; evaluation; Delphi Technique. 